\title{
Post-launch calibration-validation and data quality evaluation of SCATSAT-1
}

\author{
Raj Kumar*, Suchandra A. Bhowmick, Abhisek Chakraborty, Anuja Sharma, \\ Shweta Sharma, M. Seemanth, Maneesha Gupta, Prantik Chakraborty, \\ Jalpa Modi and Tapan Misra
}

Space Applications Centre, ISRO, Ahmedabad 380 015, India

\begin{abstract}
Here we provide a brief description of the post-launch data quality evaluation and calibration-validation chain of the SCATSAT-1, the second scatterometers mission of Indian Space Research Organisation. This chain is of absolute importance to monitor the satellite health and its impact on its measurements. It also provides us overview of the suitability of the data for various applications. The results show that the SCATSAT instrument is having nominal behaviour, the measurements are of very high quality and is comparable to the reference mission QuikSCAT. The ocean surface winds derived using SCATSAT-1 are having errors less than $1 \mathrm{~m} / \mathrm{s}$ and hence it is suitable for all operational meteorological and oceanographic applications.
\end{abstract}

Keywords: Calibration, data quality evaluation, scatterometers, validation, wind vectors.

\section{Introduction}

WIND vector observation over data-scarce oceans is the primary objective of scatterometers. It is also one of the major players for the study of polar ice and tropical vegetation. Moreover, scatterometers have a proven history of being useful for mapping the sea-ice extent right from the time of NASA's NSCAT followed by QuikSCAT. The heritage of scatterometers has been enriched by the European Space Agency's ERS-1, ERS-2 and ASCAT. Dedicated to the mission of having continuous data records over the oceans, OSCAT scatterometer on-board Oceansat-2 was launched by the Indian Space Research Organisation (ISRO) in September 2009, followed by the continuity mission of SCATSAT-1.

SCATSAT-1 was launched on 26 September 2016 from Sriharikota High Altitude Range (SHAR) using Polar Orbiting Satellite Launch Vehicle (PSLV-C35). The satellite was placed initially in a polar non-sunsynchronous orbit at $720 \mathrm{~km}$ altitude with inclination of $98^{\circ}$. Later, it was drifted into a sun-synchronous orbit at 8.45 a.m. local time after 150 days. The primary payload

\footnotetext{
*For correspondence. (e-mail: rkumar.sharma@gmail.com)
}

is a $\mathrm{Ku}$-band $(13.515 \mathrm{GHz})$ pencil-beam dual-polarized scatterometer similar to OSCAT on-board Oceansat-2, ISROs first scatterometer mission. The inner beam $\mathrm{CH}$ polarized) has $1400 \mathrm{~km}$ swath and the outer beam $(V V$ polarized) has $1800 \mathrm{~km}$ swath providing wide coverage at a nominal resolution of $25 \mathrm{~km}$ and repeat cycle of two days. Following the lineage of scatterometers, SCATSAT-1 also has similar types of application. The major applications planned under the SCATSAT-1 utilization programme of ISRO, include forecast of meteorological hazards, ocean state forecasts, monitoring crop/vegetation and polar ice sheet, soil moisture mapping, etc. Success of all these applications depends on the accuracy of measured backscattering coefficient $\left(\sigma^{0}\right)$ and continuity of data in a longer time-frame. Thus, monitoring of satellite health and time evolution of measurement errors is extremely crucial. Hence, an ideal application programme begins with the in-flight data quality evaluation (DQE) followed by calibration (CAL) of measured $\sigma^{0}$ and validation (VAL) of the final product, i.e. wind retrieved from the backscatter using a geophysical model function (GMF). DQE is an independent chain that predominantly monitors the health of the instrument in hostile space environment along with sensor performance in near realtime basis. CAL guarantees stability and precision of its measurements by quantifying the measurement errors and biases of the instrument. While VAL emphasizes the accuracy of the final wind with respect to other observations, determining its suitability for various applications at the user's end.

In the case of SCATSAT-1 raw data, i.e. level-0 and data products generated from each acquisition of SCATSAT-1 pass through the DQE system for the conformance of data quality. The key objective of DQE system is to ensure the dissemination of best quality of data to the end user. If any deviation or anomaly is seen in the sensor data or data product quality, it is reported immediately so that proper action can be taken well on time. Moreover, the DQE system is capable of generating time series of selected parameters to observe the parameter behaviour over a period of time.

CAL exercise is based on the analysis of $\sigma^{0}$. This is done to determine accuracy of the measured $\sigma^{0}$. In case 
Table 1. SCATSAT-1 data product levels

\begin{tabular}{|c|c|c|c|c|c|}
\hline Level & Description & Parameter & Special resolution & Format & $\begin{array}{c}\text { Number of } \\
\text { products per day }\end{array}$ \\
\hline Level 1B & Scan mode & $\sigma^{0}$ & $\sim 6 \mathrm{~km} \times 30 \mathrm{~km}$ & HDFS & $2 \times 14.4$ \\
\hline Level 2A & Swath grid & $\sigma^{0}$ & $25 \mathrm{~km} \times 25 \mathrm{~km}$ and & & $2 \times 2=4 \times 14.4$ \\
\hline Level 2B & & Wind velocity & $50 \mathrm{~km} \times 50 \mathrm{~km}$ & & $2 \times 2=4 \times 14.4$ \\
\hline Level 3S & Global grid & $\sigma^{0}$ & $0.5^{\circ} \times 0.5^{\circ}$ and & & 4 \\
\hline Level 3W & & Wind velocity & $0.25^{\circ} \times 0.25^{\circ}$ & & 2 \\
\hline \multirow[t]{3}{*}{ Level 4 (India, NPOLAR, SPOLAR) } & Image form & $\sigma^{0}$ & $2 \mathrm{~km} \times 2 \mathrm{~km}$ & GEOTIFF & $6+4+4$ \\
\hline & & $\gamma^{0}$ & & & $6+4+4$ \\
\hline & & BT & $6.25 \mathrm{~km} \times 6.25 \mathrm{~km}$ & & $6+4+4$ \\
\hline \multirow[t]{3}{*}{ Level 4 (full globe) } & Image form & $\sigma^{0}$ & $2 \mathrm{~km} \times 6.25 \mathrm{~km}$ & GEOTIFF & 6 \\
\hline & & $\gamma^{0}$ & & & 6 \\
\hline & & BT & $6.25 \mathrm{~km} \times 6.25 \mathrm{~km}$ & & 6 \\
\hline Level 4 (analysed winds) & Gridded wind & Wind vectors & $25 \mathrm{~km} \times 25 \mathrm{~km}$ & NETCDF & 1 \\
\hline Level (high resolution winds) & Swath grid & Wind vectors & $6.25 \mathrm{~km} \times 6.25 \mathrm{~km}$ & NETCDF & $2 \times 14.4$ \\
\hline
\end{tabular}

of scatterometers, the nominal antenna footprint on ground is an ellipse. Each footprint contains a number of independent measurements called 'slices'. In case of SCATSAT-1 the inner beam contains 9 and outer beam contains 15 slices. Analysis of slice/footprint-level $\sigma^{0}$ gives a gross idea about the presence of any attitudedependent biases in the data. It is done by analysing $\sigma^{0}$ over certain land targets which have very high volume scattering, thus offering trivial spatial-temporal variation of the measured $\sigma^{0}$. A few such targets are Amazon, Greenland, Antarctica, etc. that are historically used for calibrating scatterometers. This is called the relative calibration $^{1,2}$ and it points out any spurious anomaly in the measured $\sigma^{0}$ due to sudden change in attitude of the spaceborne instrument.

VAL is the last phase of the pre-utilization period. Under this component, retrieved wind products are co-located and compared with the same available from observations like buoys/other scatterometer missions in tandem ${ }^{3}$ and numerical models ${ }^{4}$ to determine the manifestation of error in the ultimate product of wind. This assures the wind quality for various user applications. Validation of SCATSAT-1 wind data is done with respect to in situ observations of NDBC buoys, ECMWF model winds and ASCAT wind products.

\section{Data and methods}

The entire study is based on the SCATSAT-1 data at various levels. Table 1 shows the standard data levels for SCATSAT-1. Similar to OSCAT, data from SCATSAT1 comprise four levels, viz. scan mode level-1B, grid mode level-2, global grid level-3 and high-resolution level-4 products. Level-0 represents the raw data having spacecraft ephemeris and attitude. SCATSAT-1 provides wind vector at a cell size or resolution of $25 \mathrm{~km} \times$ $25 \mathrm{~km}$.
In the DQE and CAL-VAL exercise, various levels of SCATSAT-1 data are used. The datasets are available both from National Remote Sensing Centre, India (www.nrsc.gov.in) as well as from Meteorological and Oceanographic Satellite Data Archival Centre, India (www.mosdac.gov.in). DQE example uses L0 and L1B data, CAL uses L1B and L2A data, while VAL uses L2B data. Details of the data and methods used for various phases are discussed below.

\section{Data quality evaluation}

DQE uses level-0 sensor data and level-1B data product. The sensor parameters analysed by the DQE system from level-0 data are programmable parameters like loopback calibration power, azimuth angle, pulse repetition frequency (PRF) and echo window start time, and dynamic parameters like noise and echo data. Level-0 data consist of information from digital acquisition and compression system/payload level controller (DACS/PLC)-inserted auxilary, where both static and dynamic information like noise and echo data are stored. It also contains programmable parameters useful to extract the correct record from data format. In this work nominal mode data are taken for analysis from October 2016 to December 2018. Each parameter defined above is supposed to work within a specified range which is known while designing the system. Input data dump may contain one or more orbit data, which is first cut into the orbit using pole crossing time by DQE software. DQE estimates the noise power, time, scan rate and Doppler frequency by applying the coefficients/conversion factors, and computes statistical parameters for the full dump/orbit. Further, DQE compares the quality metrics with respect to the specifications and raises alarms in near real-time if any deviation is observed. Time series obtained from the database also helps check the system stability with respect to each parameter. 
For level-1B data analysis, scan-mode data in slices and footprint at each scan in half-orbit (north to south and south to north) are used. It has both inner $(H H)$ and outer ( $V V$ ) beam data carrying 281 and 282 footprints respectively. Level-1B data product quality is observed and evaluated using automated DQE system in operations. After its launch till December 2018, there has been continuous evolution of the SCATSAT-1 processor to meet the required operational data quality.

For quality evaluation and analysis of level-1B data, quality metrics are generated based on static (geometric and constants) and dynamic (radiometric) parameters for each half-revolution (NS/SN) datum ${ }^{5}$. The static parameters include azimuth angle difference from footprint to footprint, incidence angle, Doppler frequency shift with respect to scan, range and $\mathrm{X}$-factor which were identified and observed for each half orbit acquisition in an automatic manner. The level-1B slice histograms were observed to evaluate the peak finding approach. The dynamic parameters are signal-to-noise ratio (SNR), $\sigma^{0}$ and brightness temperature (BT). All parameters were observed based on suitable categories using the flag information (land/sea; ascending/descending, inner/outer). Here the time series of quality metrics from level-1B data products of SCATSAT-1 is shown from 21 March 2018 to 15 December 2018.

Calibration: CAL uses the SCATSAT-1 slice-level $\sigma^{0}$ from L1B as well as the footprint-level $\sigma^{0}$ along with BT and SNR from L2A. The measured $\sigma^{0}$ at L1B is reported at a slice level, inner footprint of $26 \mathrm{~km} \times 46 \mathrm{~km}$ contains nine slices, while outer footprint of $31 \mathrm{~km} \times 65 \mathrm{~km}$ contains 15 slices. For the purpose of cross-calibration, L2A $\sigma^{0}$ from OSCAT on-board OceanSAT-2 and sea winds scatterometer on-board NASA's QuikSCAT is also used. Details of these two datasets are given below.

QuikSCAT: The QuikSCAT L2A data have been utilized for the purpose of cross-calibration of SCATSAT-1. NASA's QuikSCAT (1999) is a sun-synchronous satellite at an altitude of $803 \mathrm{~km}$ and at an inclination of $98.616^{\circ}$ with global coverage of $90 \%$ of ice-free ocean every day at a wind vector cell (WVC) resolution of $25 \mathrm{~km}$. It is a pencil-beam scatterometer operating at a frequency of 13.4 GHz. The incidence angles of $46^{\circ}\left(H\right.$-pol) and $54^{\circ}$ ( $V$-pol) correspond to the inner and outer beams respectively. QuikSCAT has near-repeat orbit at every 4 days with its antenna rotating at $18 \mathrm{rpm}$ asynchronous with its pulse repetition frequency (PRF) of $92.5 \mathrm{~Hz}$. Using QuikSCAT 12 individual measurements at slice level are obtained for each footprint. Each slice is typically $6 \mathrm{~km}$ long depending on the instrument mode and antenna beam, and is $25 \mathrm{~km}$ wide. Eight of these 12 slices, are reported in the L1B data as 'egg' measurements. These footprint-level data are used to generate the L2A backscatter data that are used in the present study.
Oceansat-2: These data will also be used for the prelaunch phase. OSCAT operated in Ku-band at a nominal frequency of $13.51 \mathrm{GHz}$ over a wide swath of $1400 \mathrm{~km}$. It is a pencil-beam scatterometer with a $1 \mathrm{~m}$ parabolic dish antenna with dual-feed-generating horizontal and vertical polarization beams at incidence angle of $42.66^{\circ}(H H)$ and $49.33^{\circ}(V V)$ at a scan rate of $20.5 \mathrm{rpm}$. The inner beam is horizontally polarized and the outer beam is vertically polarized. Similar to QuikSCAT, the instantaneous footprint of the scatterometer is an ellipse. The $3 \mathrm{~dB}$ footprint of the inner beam is $26 \mathrm{~km} \times 46 \mathrm{~km}$ and that of the outer beam is $31 \mathrm{~km} \times 65 \mathrm{~km}$. The nominal slice width is $8 \mathrm{~km}$ for both beams with inter-centre distance of $19 \mathrm{~km}$ along the satellite track. The L2A data of OSCAT use the inner seven slices, where the backscattering coefficients are of good quality.

Overall quality of measured $\sigma^{0}$ of SCATSAT- 1 is determined by the distribution of $\sigma^{0}$ over various slices. This slice balancing is done by constructing the histogram of $\sigma^{0}$ for each slice over the entire surface of the earth. Thus the probability of measurements over full dynamic range of $\sigma^{0}$ for all slices is similar. For perfect slice balancing, the histograms for each slice should peak at a same value of $\sigma^{0}$. Analysis of the variation of $\sigma^{0}$ over pre-decided natural terrestrial land targets is an essential component of relative calibration. A priori knowledge of these isotropic targets with large volume scattering shows that Amazon, Greenland, Rann of kutch and Antarctica are very stable targets. Difference of $\sigma^{0}$ is computed between fore and aft looks over these target to suggest look biases present between the near-simultaneous measurements by scatterometer. Differences in $\sigma^{0}$ in ascending and descending passes were similarly calculated to depict the pass biases of the instrument. Timeseries analysis of $\sigma^{0}$, SNR and BT shows the stability of the measurements and any possible amplification of noise in the instrument over time.

\section{Validation}

Level-2B wind products from SCATSAT-1 available over the satellite swaths are used for the VAL exercise. The retrieval methodology for the primary geophysical parameter, i.e. wind vectors over the ocean surface follows the heritage of Oceansat-2 (ref. 4). Minor changes in the retrieval algorithm have been done for the backscattering coefficients with low SNR values. In addition, a cubic spline-based temporal interpolation is incorporated for the numerical model winds with respect to ambiguity removal. Both these minor changes are found to improve the quality of the derived wind products. For the purpose of validation, flagged data for rain contamination, proximity to the land-masses and unavailability of the numerical model data are discarded. The following datasets have been used for the VAL exercises.

Buoy: SCATSAT-1 winds are validated with observations from 43 buoys deployed by National Data Buoy 
Centre (NDBC), 31 buoys operated by tropical atmosphere and ocean (TAO) project, four buoys operated by Prediction and Research Moored Array in the Tropical Atlantic (PIRATA) and three buoys from Research Moored Array for African Asian Australian Monsoon Analysis and Prediction (RAMA). Since scatterometer wind products are available $25 \mathrm{~km}$ away from the coast, only the offshore and deep-water buoys are selected for the validation purpose. Details of the buoys in terms of instrumentation are described earlier ${ }^{6-8}$.

Numerical weather prediction model: We have also used wind data from numerical weather prediction (NWP) model to compare SCATSAT-1-derived winds. The main purpose of such comparison is to evaluate the quality of SCATSAT-1 winds across the satellite swath. For comparison with buoys (point observation) or with ASCAT (different swath geometry), across-track validation cannot be justified. NWP analysis from National Centre for Medium Range Weather Forecasting (NCMRWF) has been used for this purpose. Details of the model used and assimilated datasets can be found in Prasad and Indira Rani ${ }^{9}$. The analyses from NCMRWF are available with spatial sampling of $0.25^{\circ}$.

ASCAT: Surface wind vectors from SCATSAT-1 are compared with those from ASCAT (on-board MetOP-A and MetOP-B) as well as with observations from buoys for the period 1 January to 31 December 2017.

For this period reprocessed (v1.1.3) SCATSAT-1 level$2 \mathrm{~B}$ data are used. ASCAT wind vector data over the ocean are available at $25 \mathrm{~km}$ horizontal resolution between $80 \mathrm{~N}$ and $80 \mathrm{~S}$. The product has been collected from EUMETSAT Ocean and Sea Ice Satellite Applications Facility (OSI SAF) of Koninklijk Nederlands Meteorologisch Instituut (KNMI), The Netherlands.

Stress-equivalent winds are better represented by scatterometer measurements, since the scatterometer measures the impact of air on water, either directly or through breaking of wind-generated waves ${ }^{10}$. However, here scatterometer winds are assumed to be equivalent neutral winds valid at a height of $10 \mathrm{~m}$ (refs 3,4$)$. True winds from buoys available at different heights are therefore corrected for the height scale using logarithmic profile estimate prior to comparison with SCATSAT-1 winds. Even NWP winds are converted to equivalent neutral winds using procedures described by Kumar et al. ${ }^{3}$ and Chakraborty et al. $^{4}$.

Collocation criteria: Scatterometer measurements and buoy observations are considered to be collocated if the distance between the centre of a WVC and the buoy location is less than $0.25^{\circ}$, and the difference in acquisition time is less than $30 \mathrm{~min}$.

For comparison with ASCAT, the same spatiotemporal windows are used. Since both the MetOP plat- forms have nearly identical equatorial crossing time to that of SCATSAT-1, a robust collocated dataset between SCATSAT-1 and ASCAT is obtained.

For collocation with NWP data, we have used a temporal window of $\pm 3 \mathrm{~h}$. This is because NWP analyses are available at $00,06,12$ and $18 \mathrm{UTC}$ only. The spatial window in this case is the same (i.e. $0.25^{\circ}$ ).

\section{Results and discussion}

\section{Data quality evaluation}

Results show consistency in sensor-related parameters monitored for the last one and half year (Table 2). There are few cases for which alerts have been given by the DQE chain whenever deviations were observed, but the percentage of such occurrences is negligible. Loopback calibration power as well as FESA telemetry power are found stable over this period. For $99.86 \%$ of datasets, noise is found within specifications (Figure 1). Eleven instances of high noise count were reported and the reason found was high solar flare during data acquisition time. The parameters PRF, delta azimuth angle and echo window start time behaved in a normal manner for all acquisitions, except for one dataset (10 November 2016), for which more than half of the orbit data was found corrupted. Junction temperature shows that the system is working well within limits, i.e. $100^{\circ}-170^{\circ} \mathrm{C}$, and following a temporal trend. One degree of increase in temperature is observed for all the junctions during the last one year, but no adverse effect is seen in data product quality (Figure 2 and Table 3). Overall, the sensor performance is found to be satisfactory, from which high-quality data products are being generated.

The level-1B data suggest that all the static parameters are stable and well within the specified range (Table $4 a$ ). The slices were analysed based on the histograms of each orbit data. Results suggest that the peak locations of 7 out of 9 (inner beam) and 13 out of 15 (outer beam) slices match, and the energy distribution is symmetric around the central slice and well within the specifications for both land and sea data. Further, dynamic parameters such as BT, SNR and $\sigma^{0}$ were observed from land and sea data on averaging over the global two days' cycle data (Table $4 b$ ). Results suggest that BT and $\sigma^{0}$ are of good quality except for seasonal variation seen over the sea. The standard deviation in $\sigma^{0}$ is around $3.7 \mathrm{~dB}$ (land) and $5.9 \mathrm{~dB}$ (sea). Table 4 shows that $K_{p}$ is well within limits of 0 to 1 showing good-quality $\sigma^{0}$. BT is stable and ranges from $233.67 \mathrm{~K}$ to $248.66 \mathrm{~K}$ over land for $H H$ and $V V$ beams respectively; while over sea it is 108.09 and $179.79 \mathrm{~K}$ for $H H$ and $V V$ beams respectively. These ranges are as expected.

\section{Calibration}

The analysis shown here corresponds to the latest version of SCATSAT-1 data, operational since March 2018. For 
SPECIAL SECTION: SCATSAT-1

Table 2. Details of parameters considered for data quality evaluation (DQE)

\begin{tabular}{|c|c|c|c|}
\hline Parameter & Specifications & Mean & Remarks \\
\hline Loopback Cal Power & 50 to $52 \mathrm{~dB}$ for $H H$ and $V V$ & $\begin{array}{l}H H 50.6(\mathrm{dBm}) \\
V V 50.3(\mathrm{dBm})\end{array}$ & $\begin{array}{l}99.88 \% \text { instances within specifications. } \\
\text { Alert issued by DQE for seven instances }\end{array}$ \\
\hline FESA telemetry power & 50 to $52 \mathrm{~dB}$ & 51.15 & $\begin{array}{l}\text { Consistence behaviour seen. Alternative Cal } \\
\text { power source, not being used for processing }\end{array}$ \\
\hline Noise (N1 and N2) & $\leq 20 \mathrm{~dB}$ & $\begin{array}{l}H H-27.75 \\
V V-27.43\end{array}$ & $\begin{array}{l}99.86 \% \text { within specifications. Alert issues for } 11 \\
\text { instances (high solar flare) }\end{array}$ \\
\hline Pulse repetition frequency (PRF) & $\begin{array}{l}192.95 \text { to } 193.05 \mathrm{~Hz} \\
\text { for } H H \text { and } V V\end{array}$ & $\begin{array}{l}H H 192.97 \mathrm{~Hz} \\
V V 192.98 \mathrm{~Hz}\end{array}$ & $\begin{array}{l}\text { Alert issued for single instance where more than } \\
\text { half orbit of data was found as corrupted }\end{array}$ \\
\hline Delta azimuth angle & $1.27^{\circ}$ & $\begin{array}{l}1.18 \text { to } 1.38^{\circ} \\
\quad \text { (latitude dependent) }\end{array}$ & Alert issued for single instance (same as PRF) \\
\hline Echo window start time & $\begin{array}{l}H H 0.0066 \text { to } 0.0069 \mathrm{sec} \\
V V 0.0081 \text { to } 0.0082 \mathrm{sec}\end{array}$ & $\begin{array}{l}H H 0.0067 \mathrm{sec} \\
V V 0.0081 \mathrm{sec}\end{array}$ & Alert issued for single instance (same as PRF) \\
\hline
\end{tabular}
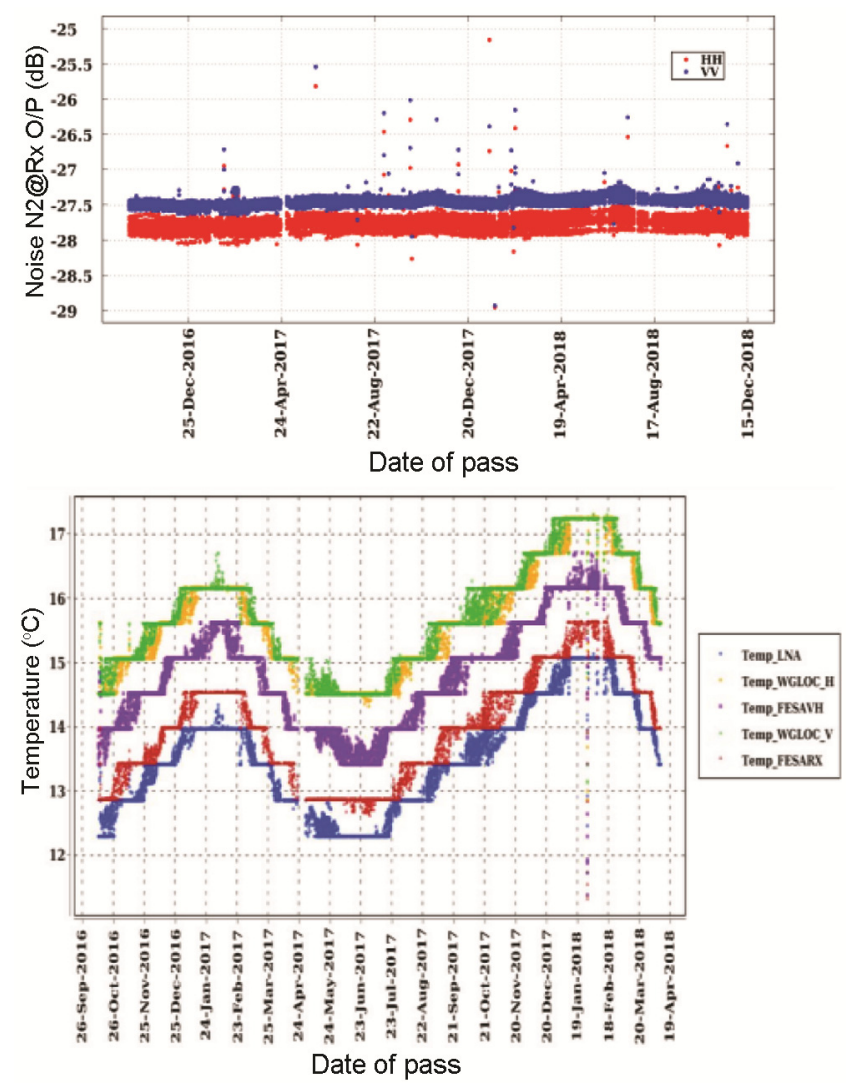

Figure 1. Time series of system noise and temperature.

the gross quality of measurement, level-1B backscatter has been analysed for several passes and histograms of $\sigma^{0}$ for each slice of inner and outer beam are constructed. Since each slice would cover all types of targets globally, the peak of histograms must match with one another. For example, Figure 3 shows histogram of $\sigma^{0}$ for nearly 100 passes from Julian day 355 to 359 of 2017 for the descending aft passes of both inner and outer beams. Clearly, the peak of global $\sigma^{0}$ is at the same place, showing a perfect slice balancing of SCATSAT-1 measurements. Likewise, fore and aft average $\sigma^{0}$ and SNR for about 64 ascending passes clearly indicates that the biases are low between the near simultaneous measurements of fore and aft (Figure 4). SNR is symmetrical about the centre slice, implying a quality measurement from SCATSAT-1. A detailed analysis of L2A $\sigma^{0}$ and BT from 1 January to 30 June 2017 is also performed. It shows that over the invariant sites, there is a trivial bias between the fore/aft looks and ascending/descending passes. Case of outer beam with $V V$ polarization is shown here. The pass and look bias for Amazon (Figure 5) implies that variation between the near simultaneous observations (fore/aft) of $\sigma^{0}$ and diurnal variability of $\sigma^{0}$ over Amazon is within $\pm 0.25 \mathrm{~dB}$, implying a consistent L2A $\sigma^{0}$ from SCATSAT-1. The time series analysis of BT of SCATSAT-1 shows a mildly rising trend along with seasonal variability of $\sigma^{0}$ (figure not shown). The difference of aft and fore average $\sigma^{0}$ for November 2009 for QuikSCAT and OSCAT has been compared with that of SCATSAT-1 (January-June 2017) over the calibration site at Greenland (Figure 6). It can be concluded that the look bias in SCATSAT-1 is much reduced compared to OSCAT, and it is almost comparable to the reference mission of QuikSCAT. Similar is the conclusion for the pass bias when compared to OSCAT and QuikSCAT.

\section{Validation}

SCATSAT-1 and ASCAT have similar equator crossingtime. Collocation of wind from the duo results in 36 million collocated wind vector pairs for the one-year period. Figure 7 shows a scatter plot of comparison between SCATSAT-1 and ASCAT-derived winds. Biases in both wind speed and direction are negligible. The root mean square (RMS) deviation in wind speed is $0.94 \mathrm{~m} / \mathrm{s}$ and that in wind direction is $15.89^{\circ}$, showing that the mission 

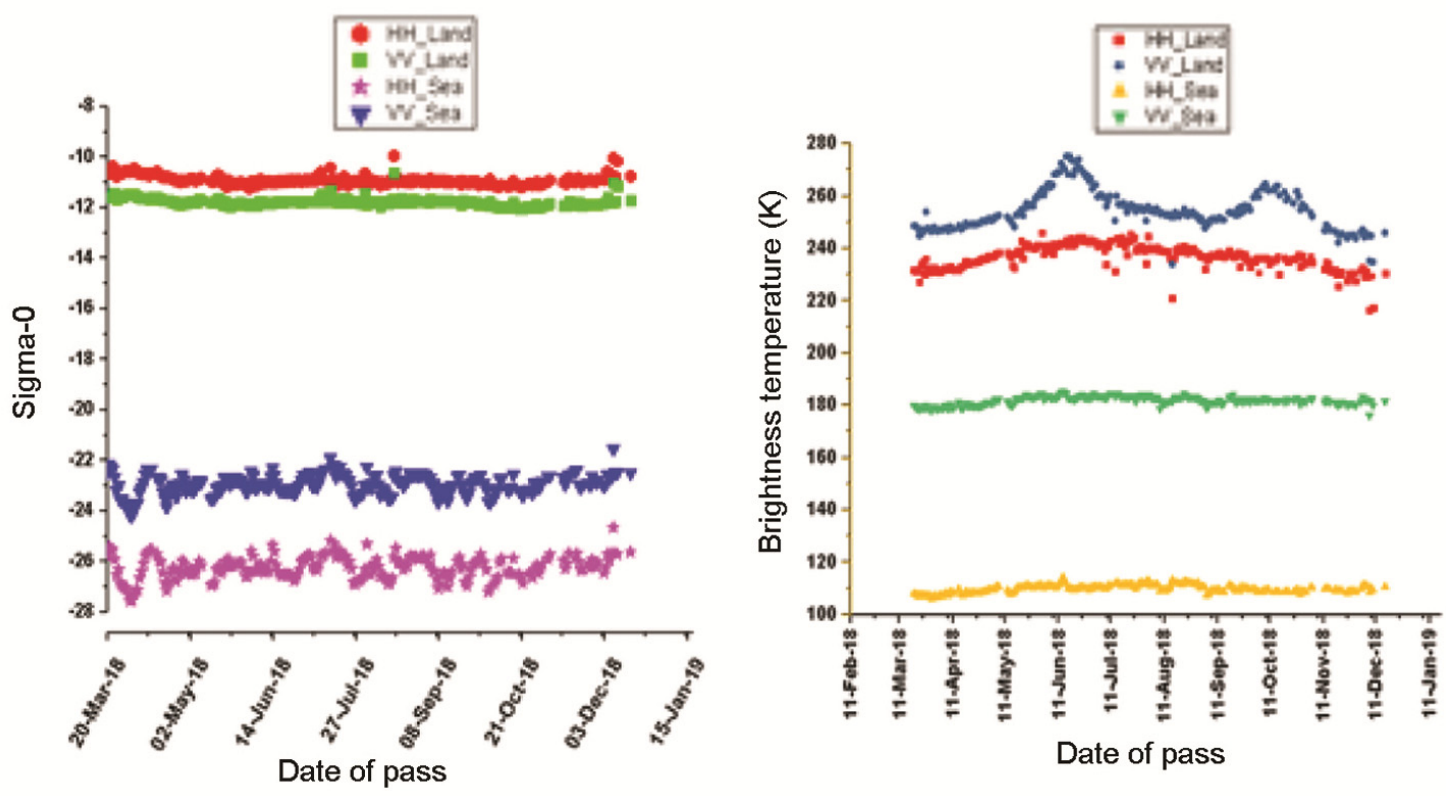

Figure 2. Time series of $\sigma^{0}$ and brightness temperature (BT).

\section{Descending-HH-Aft}

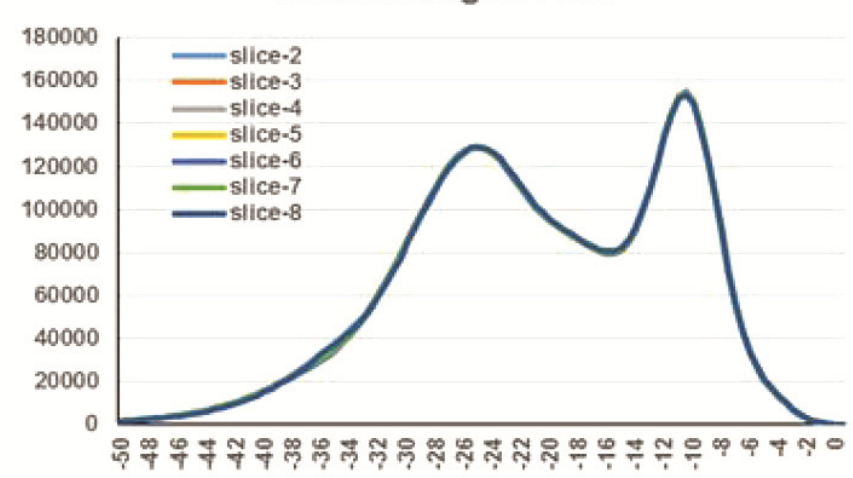

Descending-VV-Aft

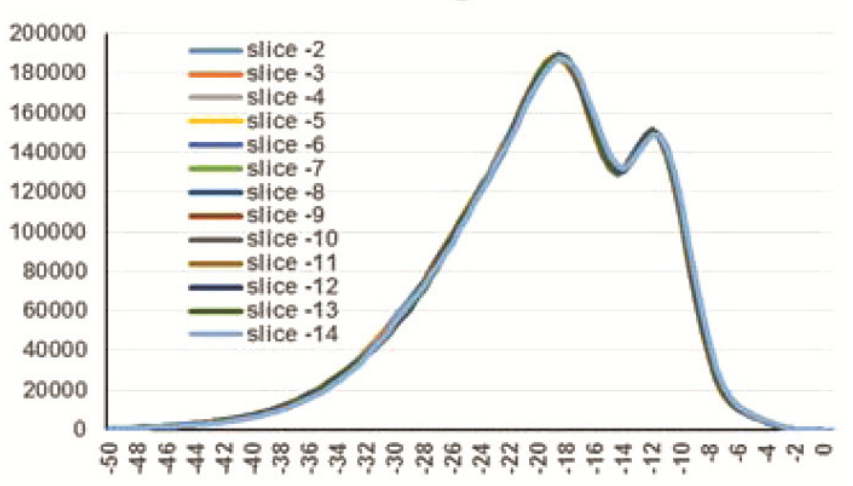

Figure 3. Histogram of inner and outer beam backscatter for each slice for the entire globe for descending aft beams.
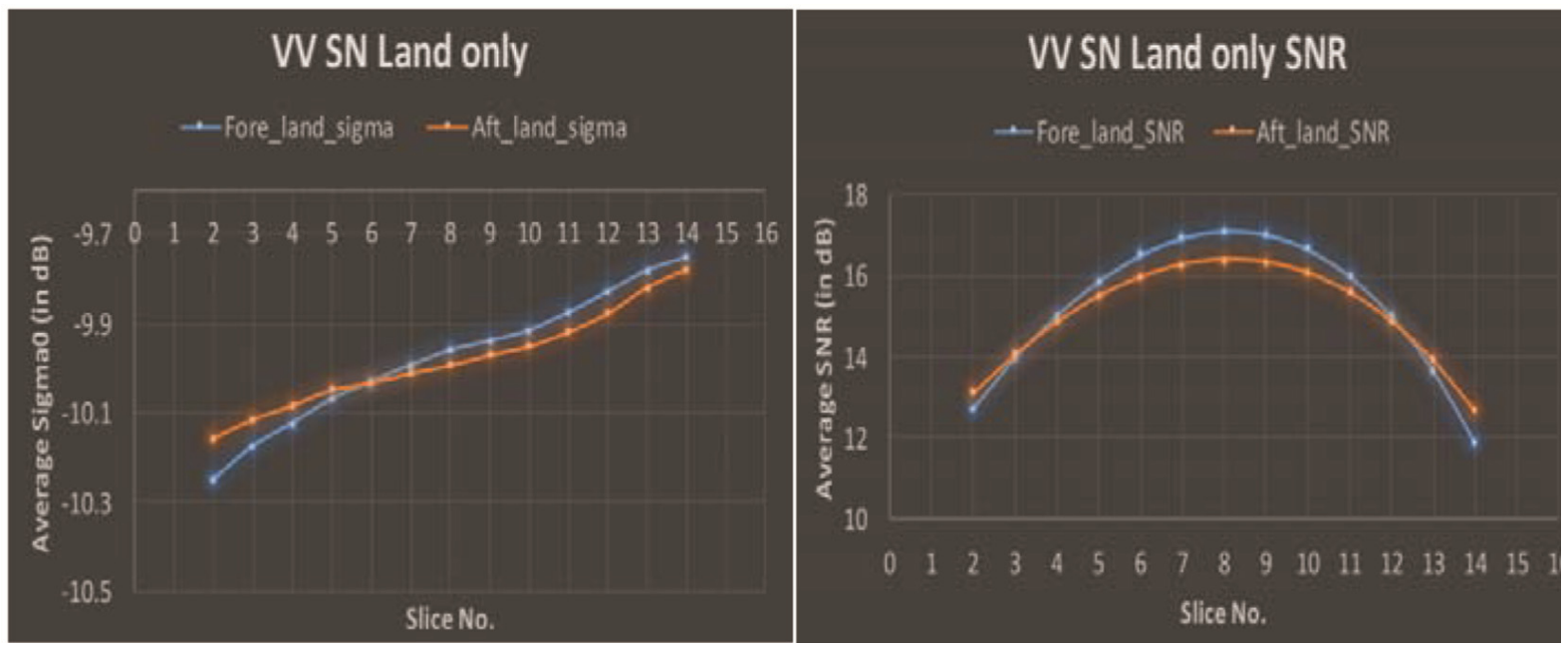

Figure 4. Variation of outer ascending beam backscatter and signal-to-noise ratio (SNR) for each slice over global land. 

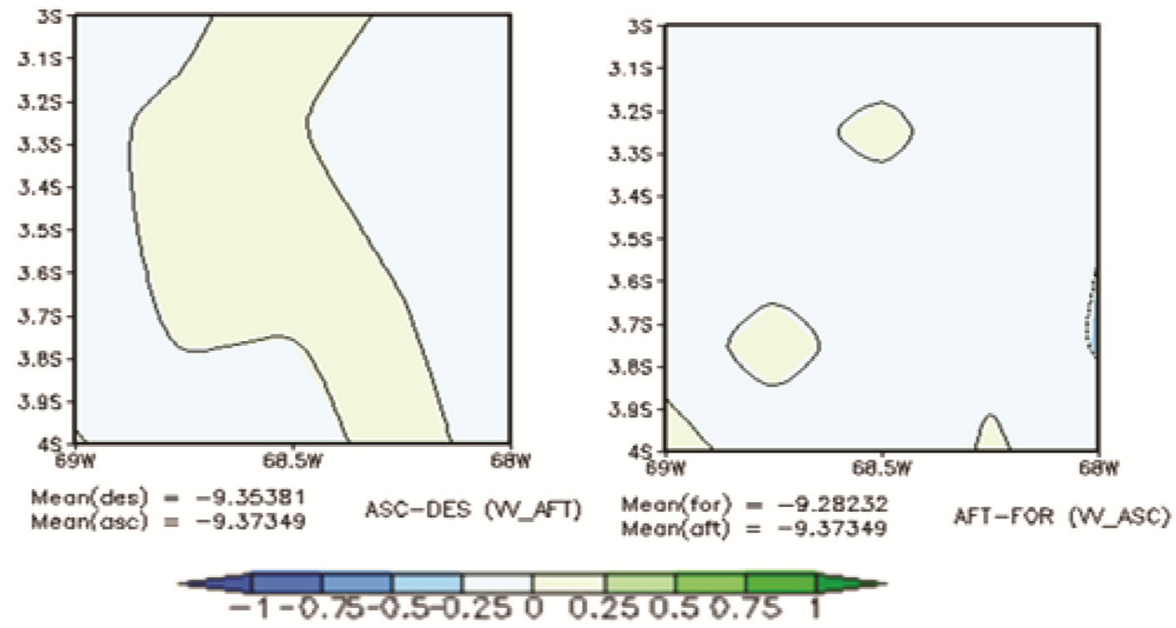

Figure 5. Pass and look bias for outer beam backscatter over the calibration site in Amazon (1 January30 June 2017).
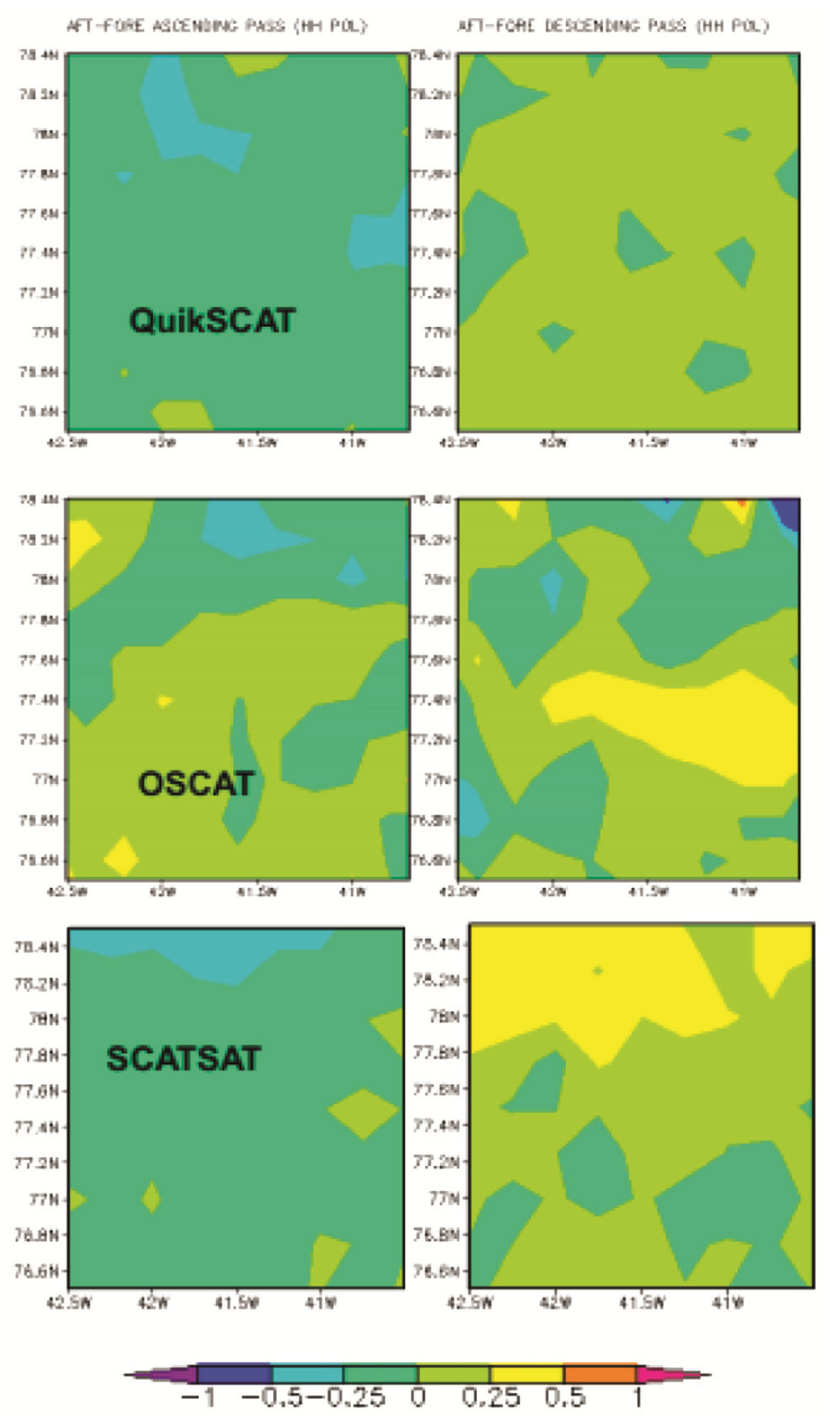

Figure 6. Comparative analysis of QuikSCAT-OSCAT-SCATSAT-1 $\sigma^{0}$ look biases over Greenland calibration site.
Table 3. Temperatures at the junctions

\begin{tabular}{lccc}
\hline & \multicolumn{3}{c}{ Temperature } \\
\cline { 2 - 4 } Junction & Minimum $\left({ }^{\circ} \mathrm{C}\right)$ & Maximum $\left({ }^{\circ} \mathrm{C}\right)$ & Mean $\left({ }^{\circ} \mathrm{C}\right)$ \\
\hline LNA & 11.38 & 15.14 & 13.53 \\
WGLOG_H & 12.85 & 17.30 & 15.64 \\
FESAV_H & 11.87 & 16.71 & 14.86 \\
WGLOG_V & 13.14 & 17.32 & 15.74 \\
FESAR_X & 11.31 & 15.68 & 13.96 \\
\hline
\end{tabular}

specification (i.e. less than $2 \mathrm{~m} / \mathrm{s}$ in speed and less than $20^{\circ}$ in direction for $3-30 \mathrm{~m} / \mathrm{s}$ wind speed range) is successfully met. Figure 8 shows the peak normalized histograms of wind speed for the collocated data. The peak-to-peak match of histograms for both SCATSAT-1 and ASCAT winds is unique, with both resembling Weibull distributions, which is typical for the marine winds. Figure 9 spatial distribution of RMS difference (RMSD) in wind speed and direction of one year. It implies that in most of regions over the global oceans, the wind speed RMSD is less than $1 \mathrm{~m} / \mathrm{s}$ and wind direction RMSD is less than $10^{\circ}$. The wind speed RMSD is more over the rain-dominated regions and over the polar seas. This implies that there is further scope of improvement in rainand ice-flagging algorithms. Wind direction RMSD is larger for rain-dominated regions and also in the Arabian Sea (dominated by wind reversal during monsoon).

To assess the quality of retrieved winds across the satellite swath, SCATSAT-1 derived winds are compared with analysed winds from NCMRWF. Figure 10 shows the across-track variation of bias and RMS in speed and direction for the collocated SCATSAT-1/NCMRWF datasets over the global oceans. The RMS error in wind speed and direction is less than $1.6 \mathrm{~m} / \mathrm{s}$ and $16^{\circ}$ respectively, for all WVCs across the swath. At the edges and at 

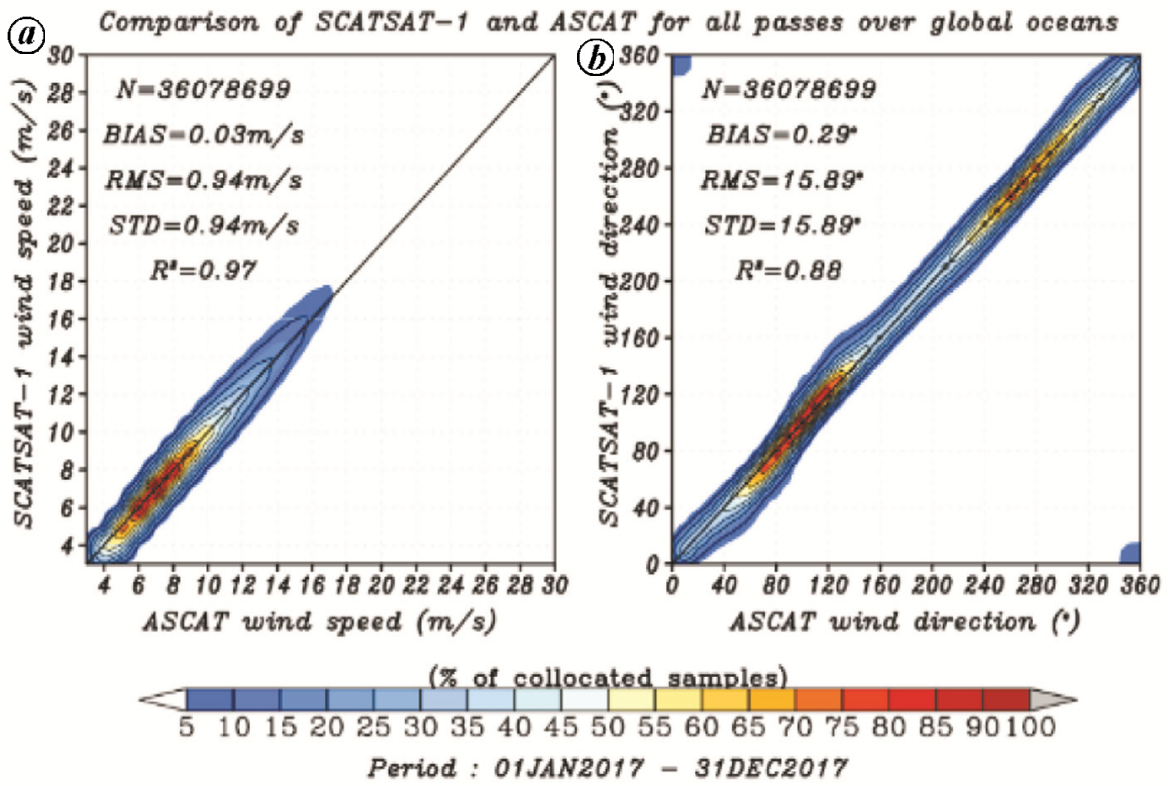

Figure 7. Scatter plot showing comparison for wind speed (a) and wind direction (b) between SCATSAT-1 and ASCAT (MetOP-A and MetOP-B).

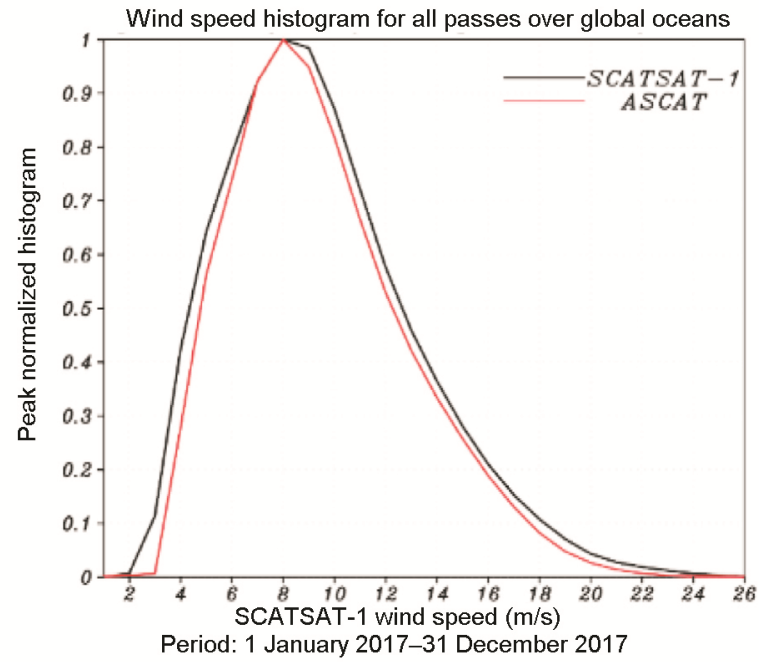

Figure 8. Peak-normalized wind speed histograms (within a bin size of $1 \mathrm{~m} / \mathrm{s}$ ) of SCATSAT-1 and ASCAT for a period of one year.

the mid-swath regions, the RMS error increases slightly. This is because at those regions, the azimuthal diversity in SCATSAT-1 observations is poor, which is a limitation for all conical scanning scatterometers. An interesting point to be noted here is that the RMS error in speed is more in comparison with NCMRWF than with ASCAT. This is mainly because time difference between SCATSAT-1 acquisition and NWP analyses can be at most $3 \mathrm{~h}$, whereas the same in case of SCATSAT- 1 and ASCAT collocation is $30 \mathrm{~min}$.

Validation with buoy observations results is an RMS error of $1.52 \mathrm{~m} / \mathrm{s}$ in speed and $16^{\circ}$ in wind direction (figure not shown). The wind speed error with buoy is larger, as the buoy represents point observations whereas SCATSAT- 1 data are averaged over an area $0.25^{\circ} \times 0.25^{\circ}$ surrounding the buoy location.

\section{Conclusion}

The SCATSAT-1 is regularly providing ocean surface winds regularly at global scales. Monitoring of sensor parameter, measurements made (like $\sigma^{0}$, SNR and BT) and henceforth retrieved final wind products is done at a regular basis in ISRO. This consists of DQE and the following CAL-VAL activities in the commissioning phase of the satellite. This study provides a glimpse of these three activities. The results clearly indicate a nominal performance of SCATSAT-1 in the space environment. The system temperature and noise are under control, except for some spooky and discrete events. The measured $\sigma^{0}$ is of extremely good quality with a perfect slicelevel balancing. The backscatter and BT are stable with a look bias and pass bias well within $\pm 0.25 \mathrm{~dB}$. The crosstrack biases are stable, less than that of OSCAT and much less than $0.5 \mathrm{~dB}$, which explicitly implies a good quality of wind for end-users. The cross-calibration with QuikSCAT and OSCAT shows that the performance of SCATSAT-1 surpasses that of OSCAT in all regards and is comparable to that of QuikSCAT. Validation of SCATSAT-1 retrieved wind vectors over the global oceans is performed against in situ wind observations from moored buoys, data from contemporary scatterometers like ASCAT and from operational NWP analyses available from NCMRWF. All these comparisons show 
SPECIAL SECTION: SCATSAT-1

Table 4. Static and dynamic parameters from L1B

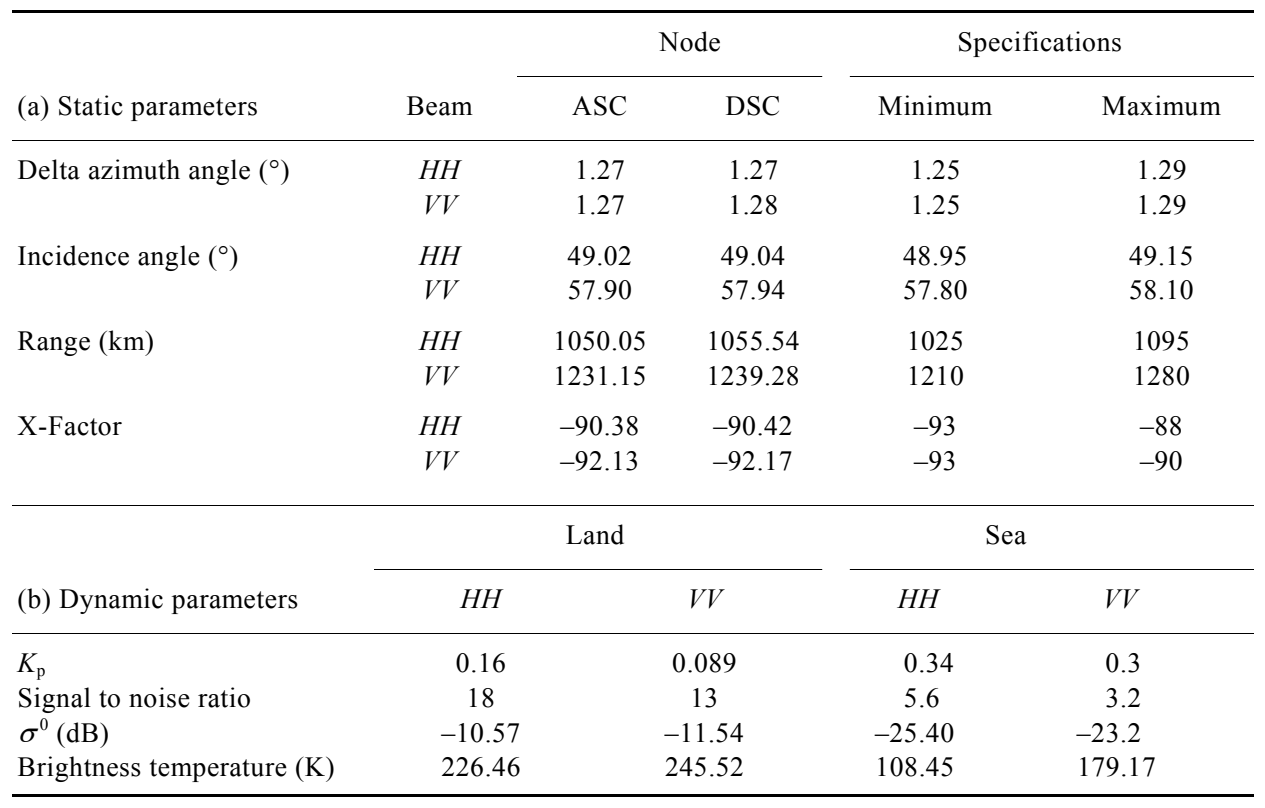
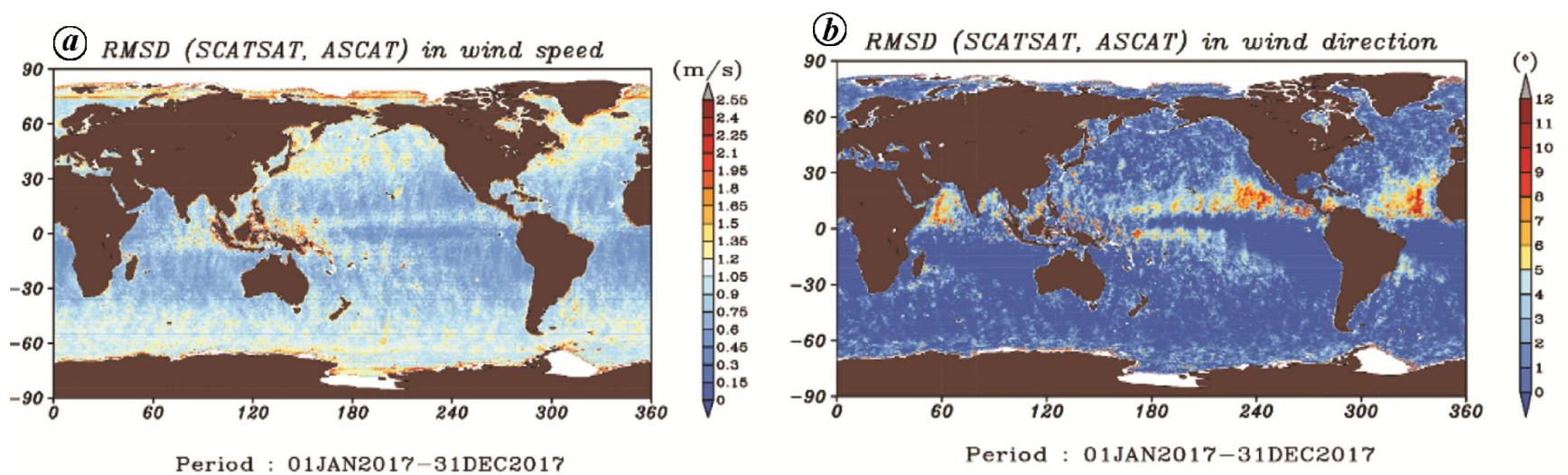

Figure 9. Spatial distribution of root mean square difference (RMSD) in wind speed (a) and wind direction (b) over the global oceans for a period of one year.

(a)

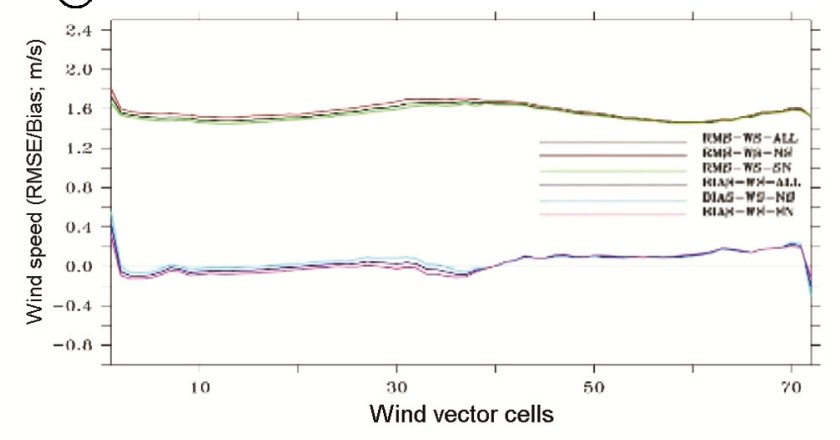

Period: 1 January 2017-31 December 2017

Figure 10. Across-track variation of root mean square (RMS) and bias for wind speed (a) and wind direction (b) for a period of one year. 
that mission specifications for SCATSAT-1 have been successfully met. This indicates that SCATSAT-1derived winds have sufficient accuracy (less than $1 \mathrm{~m} / \mathrm{s}$ in speed and less than $16^{\circ}$ in direction) required for assimilation into NWP models on operational basis.

1. Kumar, R., Bhowmick, S. A., Babu, K. N., Nigam, R. and Sarkar, A., Relative calibration of scatterometer backscattering coefficient using natural land targets - a preparatory study for OCEANSAT-2 scatterometer. IEEE Trans. Geosci. Remote Sensing, 2011, 49(6), 2268-2273.

2. Bhowmick, S. A., Kumar, R. and Kiran Kumar, A. S., Crosscalibration of the OceanSAT-2 scatterometer with QuikSCAT scatterometer using natural terrestrial targets. IEEE Trans. Geosci. Remote Sensing, 2014, 52(6), 3393-3398.

3. Kumar, R., Chakraborty, A., Parekh, A., Sikhakolli, R., Gohil, B. S. and Kiran Kumar, A. S., Evaluation of Oceansat-2-derived ocean surface winds using observations from global buoys and other scatterometers. IEEE Trans. Geosci. Remote Sensing, 2013, 51(5), 2571-2576

4. Chakraborty, A., Deb, S. K., Sikhakolli, R., Gohil, B. S. and Kumar, R., Intercomparison of OSCAT winds with numerical- model-generated winds. IEEE Geosci. Remote Sensing Lett., 2013 10(2), 260-262.

5. Gupta, M., Desai, Y. and Kartikeyan, B., Strategy for quality evaluation of OSCAT data. In Fourth International Conference of Environmental Research, Surat, Gujarat, India, 15-17 December 2011.

6. McPhaden, M. J. et al., RAMA: the research moored array for African-Asian-Australian monsoon analysis and prediction. Bull. Am. Meteorol. Soc., 2009, 90, 459-480.

7. Meindl, E. A. and Hamilton, G. D., Programs of the National Data Buoy Center. Bull. Am. Meteorol. Soc., 1992, 73(7), 985-993.

8. Bourles, B. et al., The PIRATA program history, accomplishments and future directions. Cover story. Bull. Am. Meteorol. Soc., 2008, 89(8), 1111-1125.

9. Prasad, V. S. and Indira Rani, S., Data pre-processing for NCMRWF Unified Model (NCUM): Version 2. NCMRWF research report, NMRF/RR/01/2014, 2014; http://www.ncmrwf. gov.in/ncum obstore v2.pdf

10. De Kloe, J., Stoffelen, A. and Verhoef, A., Improved use of scatterometer measurements by using stress-equivalent reference winds. IEEE J. Sel. Top. Appl. Earth, 2017, 10(5), 2340-2347; doi: 10.1109/JSTARS.2017.2685242.

doi: $10.18520 / \mathrm{cs} / \mathrm{v} 117 / \mathrm{i} 6 / 973-982$ 